Research Article

\title{
Stagnation Flow of a SWCNT Nanofluid towards a Plane Surface with Heterogeneous-Homogeneous Reactions
}

\author{
Sohail Ahmed, ${ }^{1}$ Hang Xu ${ }^{1}{ }^{1}$, and Qiang Sun $\mathbb{D D}^{2}$ \\ ${ }^{1}$ Collaborative Innovation Center for Advanced Ship and Deep-Sea Exploration (CISSE), State Key Lab of Ocean Engineering, \\ School of Naval Architecture, Ocean and Civil, Engineering, Shanghai Jiao Tong University, Shanghai 200240, China \\ ${ }^{2}$ Australian Research Council Centre of Excellence for Nanoscale BioPhotonics (CNBP), School of Science, RMIT University, \\ Melbourne, VIC 3001, Australia \\ Correspondence should be addressed to Hang Xu; hangxu@sjtu.edu.cn and Qiang Sun; qiang.sun@rmit.edu.au
}

Received 14 December 2019; Accepted 4 April 2020; Published 30 April 2020

Academic Editor: Salvatore Alfonzetti

Copyright ( 2020 Sohail Ahmed et al. This is an open access article distributed under the Creative Commons Attribution License, which permits unrestricted use, distribution, and reproduction in any medium, provided the original work is properly cited.

\begin{abstract}
The homogeneous-heterogeneous reaction in the boundary layer flow of a water-based nanofluid in the stagnation-point region of a plane surface is investigated. The type of small particles explored here is the single-walled carbon nanotubes. The homogeneous nanofluid model is employed for description of behaviours of nanofluids. Here, the homogeneous (bulk) reaction is isothermal cubic autocatalytic, while the heterogeneous (surface) reaction is single, isothermal, and first order. The steady state of this system is analysed in detail, with equal diffusion coefficients being considered for both reactants and autocatalysts. Multiple solutions of the reduced system are captured for some particular sets of physical parameters, which seem to be overlooked in all previous published works with regard to studies of homogeneous-heterogeneous reactions modeled by homogeneous nanofluid models. Besides, we discover the significant limitation of previous conclusion about that the solutions by homogeneous nanofluid flow models can be recovered from those by regular fluids.
\end{abstract}

\section{Introduction}

Chemically reacting systems in liquids or biochemical systems such as catalysis and burning admit both homogeneous and heterogeneous reactions. However, the correlation between homogeneous and heterogeneous reactions is rather complex to handle. The reaction heat that is generated or absorbed during the chemical reactions has strong influence on the flow and heat transfer in the surrounding fluid that in turn affects the local concentrations of the reactants and the products and eventually the performance of the chemical reactions.

Motivated by experimental observations of Williams et al. [1] and Song et al. [2] with regard to homogeneousheterogeneous reactions on methane/ammonia and propane oxidation over a platinum, Chaudhary and Merkin [3, 4] developed a mathematical model on homogeneous-heterogeneous reactions in which the bulk reaction is expressed by the isothermal cubic autocatalytic kinetics and the surface reaction is governed by the first-order kinetics. Xu [5] made an extension to the reaction model of Chaudhary and Merkin $[3,4]$ to introduce the effect of the homogeneousheterogeneous reactions into the energy equation.

It has been known that heat transfer rate of base fluids could be enhanced significantly as highly conductive solid particles are added into them [6-8]. For example, Choi et al. [6] discovered the prominent heat transfer enhancement of working fluid when copper nanotubes are in suspension of water. As such, if the nanoparticles are added in the working fluid to make it become a nanofluid, since the thermophysical properties of the fluid have been changed, the chemical reaction happening in that fluid can be affected. When assuming that the suspended nanoparticles are not involved in the chemical reactions of interest, $\mathrm{Xu}$ and his collaborators $[9,10]$ further extended the homogeneous-heterogeneous reaction model to nanofluids by using Buongiorno's approach [11]. Hayat et al. [12] applied the homogeneousheterogeneous reaction model to three-dimensional flow of a 
nanofluid in the suspension of carbon nanotubes over a nonlinear stretchable surface. Bachok et al. [13] studied a stagnation region flow towards a stretching sheet with homogeneous-heterogeneous reaction effects. Other works engaged in the application of the homogeneous-heterogeneous model to different flow and heat transfer problems can be found in the literature studies [14-17].

The suspended nanoparticles in nanofluids can be either metal, metal oxide, carbon nanotubes, or the combinations of them. Recently, carbon nanotubes (CNTs) have attracted much attention since they were discovered by Iijima [18] through discharging carbon electrodes to develop the new multiwalled carbon nanotubes (MWCNTs). Bethune et al. [19] then reported the development of single-walled carbon nanotubes (SWCNTs) that are structurally different from the multiwalled carbon nanotubes (MWCNTs). Since then, carbon nanotubes have become an attractive topic owing to their unique mechanical and thermal properties. Ramasubramaniam et al. [20] fabricated homogeneous single-wall carbon nanotubes (SWCNTs) for electrical conductivity applications. Xue [21] established a relation for effective thermal conductivity of carbon nanotubes based on composites. The high diffusion conductivity of the single-wall carbon nanotubes was then checked by some researchers such as Ma et al. [22], Khoshmehr et al. [23], and Park and Kim [24].

As aforementioned, chemical reactions in liquids are complex, and different reaction rates can happen even under the same conditions. From the modelling point of view, this means that the solutions of the theoretical model for the chemical reaction of interest may not be unique. Though a few studies on homogeneous-heterogeneous chemical reactions in the boundary layer flow and heat transfer based on the homogeneous nanofluid model [8] have been done, no solution multiplicities yet have been reported. Based on the discussions by Chaudhary and Merkin [3, 4] as well as Xu and his collaborators $[5,9,10]$, multiple solutions could be possible for homogeneousheterogeneous chemical reactions with the homogeneous nanofluid model. In this paper, we investigated the homogeneous-heterogeneous reactions in a nanofluid in suspension of the single-walled carbon nanotubes (SWCNTs) when equal diffusion coefficients are considered for both autocatalyst and reactants. We found the existence of solution bifurcations which, to the best of our knowledge, has not been reported before. We also testified the validation of the previous conclusion [25] on that the solutions by homogeneous nanofluid flow models can be recovered from those for regular fluids and commented that it is only valid for a few special cases. This work can be deemed as a significant improvement of previous studies on homogeneous-heterogeneous reactions in a homogeneous nanofluid.

\section{Mathematical Modeling}

2.1. Problem Description. Consider a steady boundary layer flow and heat transfer of a SWCNT-nanofluid in the stagnation-point region towards a plane surface in the presence of homogeneous-heterogeneous chemical reactions. The homogeneous-heterogeneous reaction model proposed by Chaudhary and Merkin [3, 4] is applied to describe the behaviours of chemical reactions. In this model, the homogeneous reaction takes place in the bulk, which is isothermal cubic autocatalytic and governed by

$$
A+2 B \longrightarrow 3 B, \quad \text { rate }=k_{1} a b^{2},
$$

while the heterogeneous reaction occurs on the catalyst surface, which is single, isothermal, and the first order and written by

$$
A \longrightarrow B, \quad \text { rate }=k_{s} a
$$

In the aforementioned equations, $a$ and $b$ denote concentrations of the species $A$ and $B$, respectively, and $k_{1}$ and $k_{s}$ are the homogeneous and the heterogeneous reaction rates, respectively. Note that Williams et al. [1] and Song et al. [2] have confirmed the existence of homogeneousheterogeneous reactions via experiments about methane/ ammonia and propane oxidation over platinum.

It is known that the homogeneous flow model [8] has been used to predict the behaviours of homogeneous-heterogeneous chemical reactions in nanofluids' flow. By this approach, the thermal and physical properties of a nanofluid are derived from the base fluid and the suspended solid nanoparticles [21] as

$$
\begin{aligned}
& \mu_{\mathrm{nf}}=\frac{\mu_{\mathrm{f}}}{(1-\phi)^{2.5}}, \\
& \nu_{\mathrm{nf}}=\frac{\mu_{\mathrm{f}}}{\rho_{\mathrm{nf}}} \\
& \rho_{\mathrm{nf}}=(1-\phi) \rho_{\mathrm{f}}+\phi \rho_{\mathrm{CNT}}, \\
& \left(\rho c_{p}\right)_{\mathrm{nf}}=(1-\phi)\left(\rho c_{p}\right)_{\mathrm{f}}+\phi\left(\rho c_{p}\right)_{\mathrm{CNT}}, \\
& \alpha_{\mathrm{nf}}=\frac{k_{\mathrm{nf}}}{\left(\rho c_{p}\right)_{\mathrm{nf}}} \\
& k_{\mathrm{nf}}=\frac{(1-\phi)+2 \phi\left(k_{\mathrm{CNT}} /\left(k_{\mathrm{CNT}}-k_{\mathrm{f}}\right)\right) \ln \left(\left(k_{\mathrm{CNT}}+k_{\mathrm{f}}\right) / 2 k_{\mathrm{f}}\right)}{(1-\phi)+2 \phi\left(k_{\mathrm{f}} /\left(k_{\mathrm{CNT}}-k_{\mathrm{f}}\right)\right) \ln \left(\left(k_{\mathrm{CNT}}+k_{\mathrm{f}}\right) / 2 k_{f}\right)} k_{\mathrm{f}}
\end{aligned}
$$

where $\phi$ is the volume fraction of nanoparticles, $\mu$ is the dynamic viscosity, $\nu$ is the kinematic viscosity, $\rho$ is the density, $\alpha$ is the thermal diffusivity, $c_{p}$ is the heat capacity, and $k$ is the thermal conductivity. The subscripts $\mathrm{nf}, \mathrm{f}$, and CNT denote nanofluid, fluid, and carbon nanotube (SWCNT), respectively. The thermophysical properties of water and SWCNTs used in this study are listed in Table 1.

Correspondingly, the governing equations describing the conservations of total mass, momentum, and energy, as well as chemical reaction diffusion, in the presence of singlewall carbon nanotubes (SWCNTs), in the framework of the boundary-layer approximations, are written as 
TABLE 1: Thermophysical characteristics of base fluid and SWCNTs.

\begin{tabular}{lcc}
\hline $\begin{array}{l}\text { Physical } \\
\text { characteristics }\end{array}$ & Base fluid (water) & $\begin{array}{c}\text { Nanoparticles } \\
\text { (SWCNTS) }\end{array}$ \\
\hline$\rho\left(\mathrm{k}_{\mathrm{g}} / \mathrm{m}^{3}\right)$ & 997.1 & 2600 \\
$c_{p}\left(\mathrm{~J} / \mathrm{k}_{\mathrm{g}} \mathrm{k}\right)$ & 4179 & 425 \\
$k(\mathrm{~W} / \mathrm{mk})$ & 0.613 & 6600 \\
\hline
\end{tabular}

$$
\begin{aligned}
& \frac{\partial u}{\partial x}+\frac{\partial v}{\partial y}=0, \\
& u \frac{\partial u}{\partial x}+v \frac{\partial u}{\partial y}=v_{\mathrm{nf}} \frac{\partial^{2} u}{\partial y^{2}}+u_{e} \frac{\partial u_{e}}{\partial x}, \\
& u \frac{\partial T}{\partial x}+v \frac{\partial T}{\partial y}=\alpha_{\mathrm{nf}} \frac{\partial^{2} T}{\partial y^{2}}, \\
& u \frac{\partial a}{\partial x}+v \frac{\partial a}{\partial y}=D_{A} \frac{\partial^{2} a}{\partial y^{2}}-k_{1} a b^{2}, \\
& u \frac{\partial b}{\partial x}+v \frac{\partial b}{\partial y}=D_{B} \frac{\partial^{2} b}{\partial y^{2}}+k_{1} a b^{2},
\end{aligned}
$$

subject to the boundary conditions

$$
\begin{aligned}
u & =0, v=0, \\
T & =T_{w}, \\
D_{A} \frac{\partial a}{\partial y} & =-D_{B} \frac{\partial b}{\partial y}=k_{s} a, \\
\text { at } y & =0, \\
u & =u_{e}=c x, \\
T & \longrightarrow T_{\infty}, \\
a & \longrightarrow a_{\infty}, \\
b & \longrightarrow 0, \\
\text { as } y & \longrightarrow \infty,
\end{aligned}
$$

where $u$ and $v$ are the fluid velocity components in the $x$ and $y$-directions, respectively, $u_{e}$ is the incoming velocity of external flow, $D_{A}$ and $D_{B}$ are the diffusion coefficient of species $A$ and $B$, respectively, $T_{w}$ is the surface temperature, $T_{\infty}$ is the reference temperature, $c$ is a constant, and $a_{\infty}$ is the concentration of species $A$ at the far field.

2.2. Scaling Transformation. In the studies of flow and heat transfer in the boundary layers, it is a common practice to introduce the boundary layer assumptions [26] to reduce the governing partial differential equations to ordinary differential equations via similarity transformation. The resulting equations contain self-similar solutions which are similar to themselves as independent and dependent variables are appropriately scaled.

We utilize the same idea to handle the problem considered in this work. In doing so, we introduce the following similarity variables:

$$
\begin{aligned}
\eta & =\left(\frac{c}{v_{\mathrm{f}}}\right)^{1 / 2} y, \\
u & =c x f^{\prime}(\eta), \\
v & =-\left(c v_{\mathrm{f}}\right)^{1 / 2} f(\eta), \\
\theta(\eta) & =\frac{T-T_{\infty}}{T_{w}-T_{\infty}}, \\
\psi(\eta) & =\frac{a}{a_{\infty}} \\
\chi(\eta) & =\frac{b}{a_{\infty}} .
\end{aligned}
$$

Substituting the similarity variables (6) into equation (4), continuity equation (4a) is automatically satisfied, and the rest equations are reduced to

$$
\frac{1}{(1-\phi)^{2.5}\left(1-\phi+\phi \rho_{\mathrm{CNT}} / \rho_{\mathrm{f}}\right)} f^{\prime \prime \prime}+f f^{\prime \prime}-\left(f^{\prime}\right)^{2}+1=0
$$

$$
\begin{aligned}
& \frac{1}{\left[1-\phi+\phi\left(\rho c_{p}\right)_{\mathrm{CNT}} /\left(\rho c_{p}\right)_{\mathrm{f}}\right]} \frac{k_{\mathrm{nf}}}{k_{\mathrm{f}}} \theta^{\prime \prime}+\operatorname{Pr} f \theta^{\prime}=0, \\
& \frac{1}{\mathrm{Sc}_{A}} \psi^{\prime \prime}+f \psi^{\prime}-K \psi \chi^{2}=0, \\
& \frac{\epsilon}{\mathrm{Sc}_{B}} \chi^{\prime \prime}+f \chi^{\prime}+K \psi \chi^{2}=0,
\end{aligned}
$$

subject to the boundary conditions

$$
\begin{aligned}
f(0) & =0, \\
f^{\prime}(0) & =0, \\
\theta(0) & =1, \\
\psi^{\prime}(0) & =-\epsilon \chi^{\prime}(0)=K_{s} \psi(0), \\
f^{\prime}(\infty) & =1, \\
\theta(\infty) & =0, \\
\psi(\infty) & =1, \\
\chi(\infty) & =0,
\end{aligned}
$$

where $\operatorname{Pr}=\nu_{\mathrm{f}} / \alpha_{\mathrm{f}}$ is the Prandtl number, $K=k_{1} a_{\infty}^{2} / c$ is the strength coefficient of the homogeneous reaction, $\mathrm{Sc}_{A}=v_{\mathrm{f}} / D_{A}$ and $\mathrm{Sc}_{B}=\nu_{\mathrm{f}} / D_{B}$ are the Schmidt numbers, $\epsilon=D_{B} / D_{A}$ is the ratio between the diffusion coefficients, and $K_{s}=k_{s}\left(c / \nu_{\mathrm{f}}\right)^{-1 / 2} / D_{A}$ is the strength coefficient due to the heterogeneous reaction. Note that, here, all those physical parameters are defined based on the properties of the base fluid so that the influence of nanoparticles can be clearly reflected.

In many practical applications, the diffusion of species $A$ and $B$ is in comparable sizes, which indicates that the diffusion coefficients $D_{A}$ and $D_{B}$ can be deemed as equal [3]. As a result, we are able to choose $\epsilon=1$. In this case, the following relation holds: 


$$
\psi(\eta)+\chi(\eta)=1
$$

Using equations (9), (7c), and (7d) which are combined to obtain

$$
\frac{1}{\mathrm{Sc}_{A}} \psi^{\prime \prime}+f \psi^{\prime}-K \psi(1-\psi)^{2}=0
$$

2.3. Remarks on the Homogeneous Flow Model. It is worth mentioning to this end that Magyari [25] once argued that the solutions obtained by the homogeneous nanofluid flow model could be recovered by the corresponding regular fluid's results, and no further efforts need to be paid for investigation of homogeneous modelling nanofluid flows. Here, we testify the validity of his conclusion via equations (7a), (7b), and (10). In doing so, we introduce the following scaling transformations:

$$
\begin{aligned}
f(\eta) & =\lambda_{f} F(\xi), \\
\theta(\eta) & =\lambda_{\theta} \Theta(\xi), \\
\psi(\eta) & =\lambda_{\psi} \Psi(\xi), \\
\eta & =\lambda_{\eta} \xi .
\end{aligned}
$$

where $\lambda_{\mathrm{f}}, \lambda_{\theta}, \lambda_{\psi}, \lambda_{\chi}$, and $\lambda_{\eta}$ are positive constants.

Substituting equation (11) into equations (7a), (7b), and (10), we obtain

$$
\frac{\lambda_{f} \lambda_{\eta}^{3}}{(1-\phi)^{2.5}\left(1-\phi+\phi \rho_{\mathrm{CNT}} / \rho_{\mathrm{f}}\right)} F^{\prime \prime \prime}+\lambda_{f}^{2} \lambda_{\eta}^{2} F F^{\prime \prime}-\lambda_{f}^{2} \lambda_{\eta}^{2}\left(F^{\prime}\right)^{2}+1=0,
$$

$$
\frac{\lambda_{\eta}}{\left[1-\phi+\phi\left(\rho c_{p}\right)_{\mathrm{CNT}} /\left(\rho c_{p}\right)_{\mathrm{f}}\right]} \frac{k_{\mathrm{nf}}}{k_{\mathrm{f}}} \Theta^{\prime \prime}+\lambda_{f} \operatorname{Pr} F \Theta \prime=0,
$$

$$
\frac{\lambda_{\eta}^{2}}{\mathrm{Sc}_{A}} \Psi^{\prime \prime}+\lambda_{f} \lambda_{\eta} f \Psi \prime-K \Psi\left(1-2 \lambda_{\psi} \Psi+\lambda_{\psi}^{2} \Psi^{2}\right)=0 .
$$

To resume the governing equations to Newtonian fluid's ones, it must hold

$$
\begin{aligned}
& \frac{\lambda_{\mathrm{f}} \lambda_{\eta}^{3}}{(1-\phi)^{2.5}\left(1-\phi+\phi \rho_{\mathrm{CNT}} / \rho_{\mathrm{f}}\right)}=\lambda_{f}^{2} \lambda_{\eta}^{2}=1, \\
& \frac{\lambda_{\eta}}{1-\phi+\phi\left(\rho c_{p}\right)_{\mathrm{CNT}} /\left(\rho c_{p}\right)_{\mathrm{f}}} \frac{k_{\mathrm{nf}}}{k_{\mathrm{f}}}=\lambda_{f}, \\
& \lambda_{\eta}^{2}=\lambda_{\mathrm{f}} \lambda_{\eta}=1=\lambda_{\psi}=\lambda_{\psi}^{2} .
\end{aligned}
$$

From equation (13a), we obtain

$$
\begin{array}{r}
\lambda_{\mathrm{f}}=\left[\frac{1}{(1-\phi)^{2.5}\left(1-\phi+\phi \rho_{\mathrm{CNT}} / \rho_{\mathrm{f}}\right)}\right]^{1 / 2}, \\
\lambda_{\mathrm{f}} \lambda_{\eta}=1 .
\end{array}
$$

However, from equation (13c), we have

$$
\lambda_{\mathrm{f}}=\lambda_{\eta}=\lambda_{\psi}=1 \text {. }
$$

If only the flow field is considered, then the fixed relation between homogeneous modelling nanofluid and regular fluid's solutions is readily discovered from correlation (14). However, as multiple physical fields are taken into account, such fixed relation is no longer in existence. This is to say, it is impossible to recover homogeneous modelling nanofluid's solutions via simple scaling transformations. Therefore, we are able to conclude that Magyari's comment [25] is only available for a few specific problems. For most fluid flow problems, especially those involving into multiphysical fields, the homogeneous nanofluid model could not be replaced by scaling transformations so that it plays a significant role on investigation of flow behaviours of dilute nanofluids.

2.4. Computational Method. Solutions of governing equations (7a), (7b), and (10) subject to the boundary conditions (8) are solved by means of the shooting technique [27]. The integration distances are chosen from a range of values such as $\eta_{\infty}=20,30,40$ with the step size being set to $\Delta \eta=0.005,0.01,0.05$, respectively. If all computational results for a prescribed set of physical parameters with different integration distances and step sizes remain unchanged, then we think the solutions are stable. In this way, it is found that, for $\eta_{\infty}=20$ and $\Delta \eta=0.01$, the stability of multiple solutions can be kept. The Newtonian iteration is executed with the convergence criteria of $1.0 \times 10^{-8}$ for all considered cases to examine the accuracy of results.

\section{Solution Dependence on $K$}

It has been already known from Chaudhary and Merkin [3] that the strength coefficients of homogeneous and heterogeneous reactions are key factors to generate solution bifurcations. We therefore testify the roles of $K$ and $K_{s}$ on the solution behaviours with various nanoparticle volume fractions. In our computation, the Prandtl number is fixed, $\operatorname{Pr}=6.8$, for the base fluid (water) throughout the study. Since the homogeneous nanofluid model is only suitable for the dilute nanofluid, the range of the volume fraction of nanoparticles is chosen from 0 to $0.2(0 \leq \phi \leq 0.2)$. Particularly, from the definitions of nanofluids' parameters denoted in equation (3), it is readily known that the case $\phi=0$ corresponds to the regular fluid.

3.1. Solutions for Small and Moderate K. We start our discussion by considering the variation of $\psi(0)$ due to the jointly effects of $K$ and $K_{s}$ in the presence of SWCNT nanoparticles. Clearly, the multiple solutions still exist as the nanoparticles are considered. For example, for $\phi=0.1$ and $\mathrm{Sc}_{A}=1$, multiple solutions are found for $K_{s}=0.025$ and $K_{s}=0.05$ as $K$ in particular regions. However, for $K_{s}=0.1$, only unique solution can be found, as shown in Figure 1. It is also observed from this figure that the hysteresis point is obtained for the decreasing values of $K_{s}$ (say $K_{S}^{H}$ ). For all values, $K_{s}<K_{S}^{H}$, multiple solution are possible. 


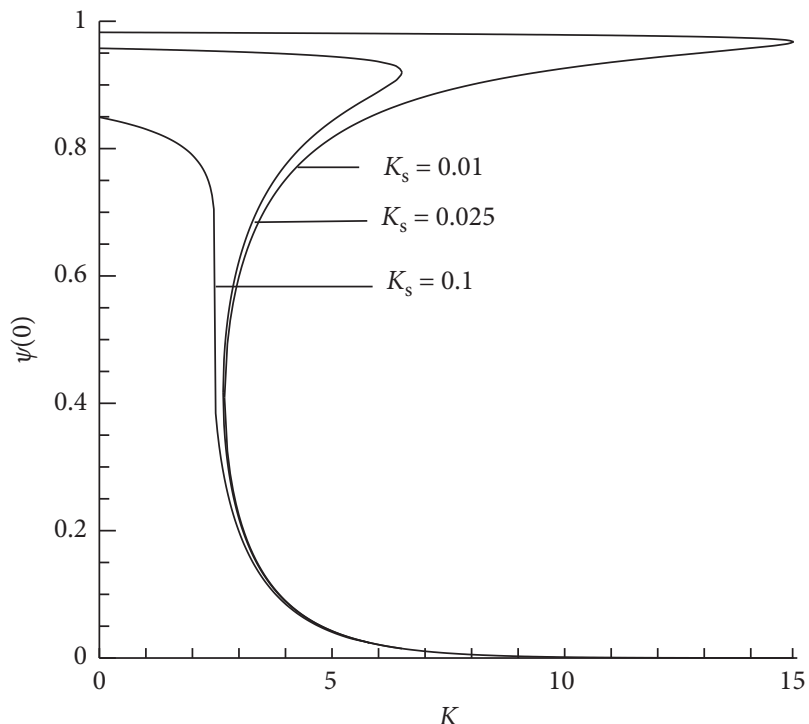

Figure 1: $\psi(0)$ against $K$ for some values of $K_{s}$ with $\operatorname{Pr}=6.8$, $\phi=0.1$, and $\mathrm{Sc}_{A}=1.0$.

The bifurcation points for possible ranges of $K$ are listed in Table 2. It is seen in this table that multiple solutions are found in the ranges $K_{\min } \leq K \leq K_{\max }$ for $K_{s}=0.025$ and $K_{\min } \leq K \leq K_{\max }$ for $K_{s}=0.05$, while only one unique solution is discovered for $K_{s}=0.1$.

We then consider the effect of the nanoparticle volume fraction $\phi$ on $\psi(0)$ for different values of $K$. In doing so, we choose $K_{s}=0.025, \operatorname{Pr}=6.8$, and $\mathrm{Sc}_{A}=1$ as an illustrative case where multiple solutions are available. As shown in Figure 2, as $\phi$ increases, the bifurcation points for both maximum and minimum shift towards the left side. This indicates that the addition of nanoparticles is helpful to keep the stability of chemical reaction for large $K$, while it could lead to the instability of chemical reaction for small $K$. The maximum and minimum points for solution multiplicities are listed in Table 3. It is clearly shown that multiple solution regions are $2.723 \leq K \leq 6.738$ for $\phi=0,2.659 \leq K \leq 6.505$ for $\phi=0.1$, and $2.568 \leq K \leq 6.174$ for $\phi=0.2$.

The influence of $K_{s}$ on $\psi(0)$ for different values of $K$ at $\phi=0.1, \mathrm{Sc}_{A}=1$, and $\operatorname{Pr}=6.8$ is presented in Figure 3 . It is observed that only one unique solution exists as the homogeneous reaction rate $K=2$, while multiple solutions are captured for $K=4$ and $K=8$ provided that $K_{s}$ is properly chosen. It is worth mentioning that, in our model assumption, the heterogeneous reaction rate $K_{s}$ is expected to be always positive. In the meantime, $\psi(\eta)$ has to be equal or greater than zero and less than or equal to 1 (owing to $a \leq a_{\infty}$ ). This means that all solutions for $K_{s}<0$ and $\psi>1$ (or $\psi<0)$ are physically meaningless. Particularly, the system can keep the self-sustained surface reaction without the homogeneous reaction being involved $(K=0)$ when nanoparticles are appended. As the hysteresis point is reached for increasing value of $K$ (say $K^{H}$ ), multiple solutions are possible for all values of $K>K^{H}$. Figure 4 shows the effect of $K_{s}$ on $\psi(0)$ for some values of nanoparticle volume fraction $\phi$. From the figure, it is observed that the variation of
TABLe 2: Minimum and maximum values (say $K_{\min }$ and $K_{\max }$ ) of $K$ at several prescribed values of $K_{s}$ in the case of $\phi=0.1, \mathrm{Sc}_{A}=1.0$, and $\mathrm{Pr}=6.8$.

\begin{tabular}{lccc}
\hline Bifurcation point & $K_{s}=0.01$ & $K_{s}=0.025$ & $K s=0.1$ \\
\hline$K_{\min }$ & 2.696 & 2.659 & - \\
$K_{\max }$ & 14.922 & 6.505 & - \\
\hline
\end{tabular}

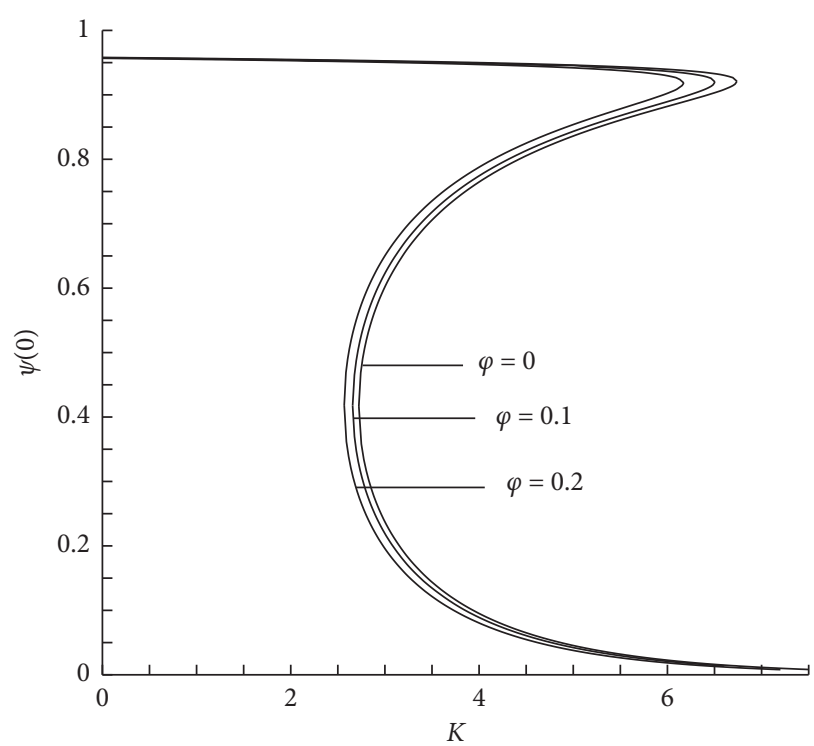

Figure 2: $\psi(0)$ against $K$ for some values of $\phi$ with $\operatorname{Pr}=6.8$, $K_{s}=0.025$, and $\mathrm{Sc}_{A}=1.0$.

Table 3: Minimum and maximum values of $K$ at several prescribed values of $\phi$ in the case of $K_{s}=0.025, \mathrm{Sc}_{A}=1.0$, and $\operatorname{Pr}=6.8$.

\begin{tabular}{lccc}
\hline Bifurcation point & $\phi=0$ & $\phi=0.1$ & $\phi=0.2$ \\
\hline$K_{\min }$ & 2.723 & 2.659 & 2.568 \\
$K_{\max }$ & 6.738 & 6.505 & 6.174 \\
\hline
\end{tabular}

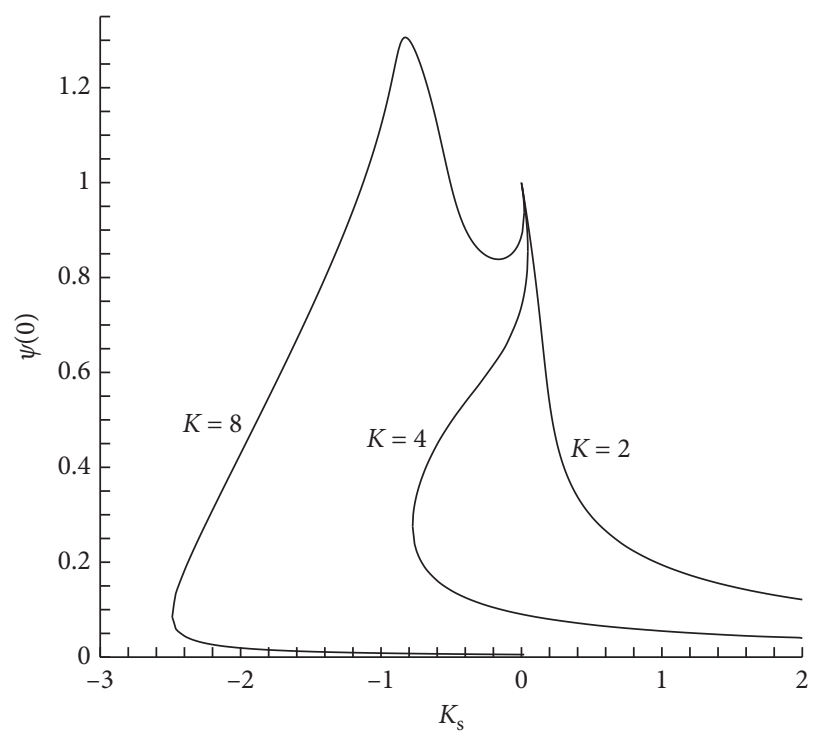

Figure 3: $\psi(0)$ against $K_{s}$ for some values of $K$ with $\operatorname{Pr}=6.8$, $\phi=0.1$, and $\mathrm{Sc}_{A}=1.0$. 


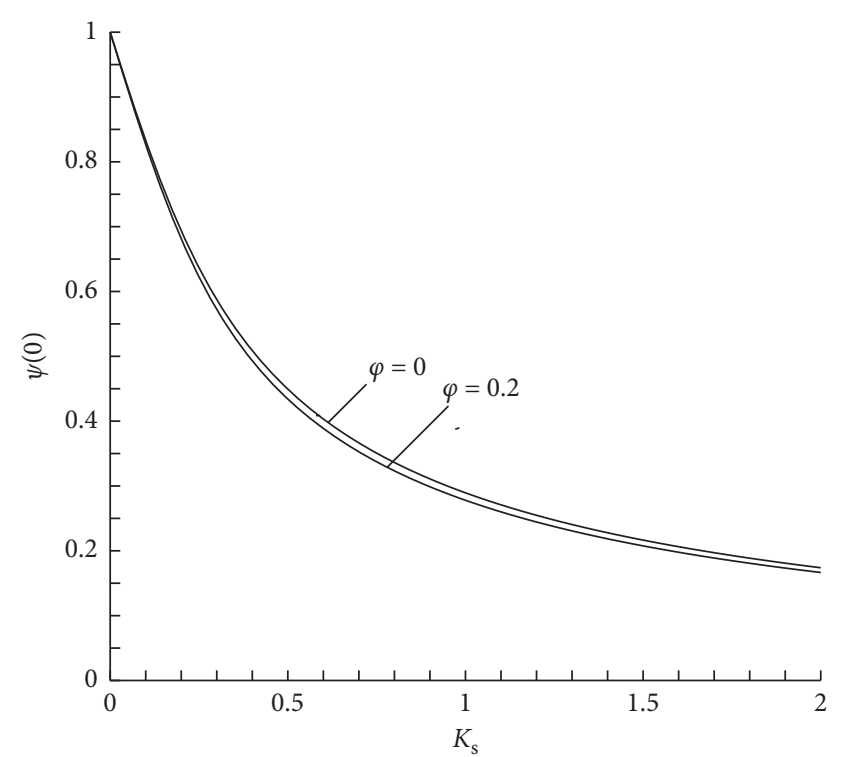

Figure 4: $\psi(0)$ against $K_{s}$ for some values of $\phi$ with $\operatorname{Pr}=6.8, K=1$, and $\mathrm{Sc}_{A}=1.0$.

$\phi$ could not alter the behavior of solution multiplicity. In other words, the existence of multiple solutions is not affected by changes of nanoparticle volume fraction. The role of the nanoparticle volume fraction is that it can accelerate or postpone the chemical reaction rate.

3.2. Solutions for Large K. It has been reported by Chaudhary and Merkin [3] that there is a shear-layer-type structure developed by the concentration profile $\psi(\eta)$ as $K$ continuously increases. The similar structure is found to be still in existence when nanofluids are considered. As shown in Figure 5(a), the reaction gradually compacts to a relatively thin region that increasingly departs from the surface as $\phi$ evolves. This figure also shows that, as $\phi$ enlarges, the thickness of the fully reacted-state region grows larger, which leads to the thickness of the unreacted-state region which becomes thinner.

From equation (10), it is expected that the asymptotic behavior for large $K$ is $K_{s}$ in the order of $O(1)$. In the reaction zone, this equation suggests

$$
\eta=R(K)+K^{-1 / 2} Y
$$

where $R(K)$ is a constant and $Y$ denotes large $\eta$. Here, the reaction front has a thickness of $O\left(K^{-1 / 2}\right)$ and is centered on $\eta=R(K) \gg 1$. Therefore, $R(K)$ is expanded as

$$
R(K)=R_{0} K^{-1 / 2}+R_{1}+\cdots, \quad \text { as } K \longrightarrow \infty,
$$

where $R_{0}, R_{1}, \cdots$, are constants to be determined.

Using equation (16) in equation (10), the following equation is obtained:

$$
\frac{1}{\mathrm{Sc}_{A}} \psi^{\prime \prime}+\left(R+K^{-1 / 2} Y-\Delta\right) \psi \prime-K \psi(1-\psi)^{2}=0
$$

subject to the boundary condition

$$
\begin{aligned}
& \psi \longrightarrow 1, \\
& Y \longrightarrow \infty .
\end{aligned}
$$

Here, the prime denotes the differentiation with respect to $Y$.

Equation (18) is expected to have a solution of the form

$$
\psi(Y ; K)=\psi_{0}(Y)+K^{-1 / 2} \psi_{1}(Y)+\cdots .
$$

Substituting equation (20) into equation (18) and then omitting the high-order terms, at the first-order, it reads

$$
\frac{1}{\mathrm{Sc}_{A}} \psi_{0}^{\prime \prime}+R_{0} \psi_{0}^{\prime}-\psi_{0}\left(1-\psi_{0}\right)^{2}=0
$$

Taking boundary condition (19) into account, it is expected that $\psi_{0}$ is exponentially small in $K$ in the inner wall region such that

$$
\psi_{0} \longrightarrow 0, \quad \text { as } Y \longrightarrow-\infty
$$

As briefly discussed in [28], equation (21) subject to boundary condition equation (22) is the cubic-Fisher wave equation that is essentially an eigenvalue problem for $R_{0}$. Its solution has exponential decaying property at infinity as expected. The reason is that the solutions to equation (21) have algebraic properties of $O\left(Y^{*}\right)$ as $Y \longrightarrow \infty$, where $Y^{*}=Y \mathrm{Sc}_{A}^{1 / 2}$, which in turn bring about the perturbation of $O\left(R e^{-1 / 2}\right)$ to $\psi$ in the external flow.

Using the method proposed by Billingham and Nadeem [28], the unique solution for equation (21) is given as

$$
\begin{aligned}
& R_{0}=\frac{1}{\sqrt{2 \mathrm{Sc}_{A}}} \\
& \psi_{0}=\left[1+\exp \left(-\frac{Y^{*}}{\sqrt{2}}\right)\right]^{-1} .
\end{aligned}
$$

At $O\left(K^{-1 / 2}\right)$, it holds

$$
\psi_{1}+\frac{1}{\sqrt{2}} \psi_{1}^{\prime}-\left(1-4 \psi_{0}+3 \psi_{0}^{2}\right) \psi_{1}=\left(\Delta-R_{1}\right) \sqrt{S c_{A}} \psi_{0}^{\prime}
$$

subject to the boundary conditions

$$
\psi_{1} \longrightarrow 0, \quad \text { as }|Y| \longrightarrow \infty,
$$

where prime denotes the differentiation with respect to $Y^{*}$.

Since equation (24) includes a complementary function $\psi_{0}^{\prime}$ which satisfies the required boundary conditions, an additional boundary condition has to be introduced to give a solution to the full equation. To meet this requirement, the following correlation is introduced (refer to [3]):

$$
\sqrt{\mathrm{Sc}_{A}}\left(\Delta-R_{1}\right) \int_{-\infty}^{\infty} \psi_{0}^{\prime 2} \exp \left(\frac{Y^{*}}{\sqrt{2}}\right) \mathrm{d} Y^{*}=0,
$$

which gives $R_{1}=\Delta$. Therefore, $R(K)$ can be expressed as

$$
R(K)=\frac{K^{1 / 2}}{\sqrt{2 S c_{A}}}+\Delta+\cdots, \quad \text { as } K \longrightarrow \infty .
$$

However, as concluded by Chaudhary and Merkin [3], equation (27) is not a complete solution since the condition 


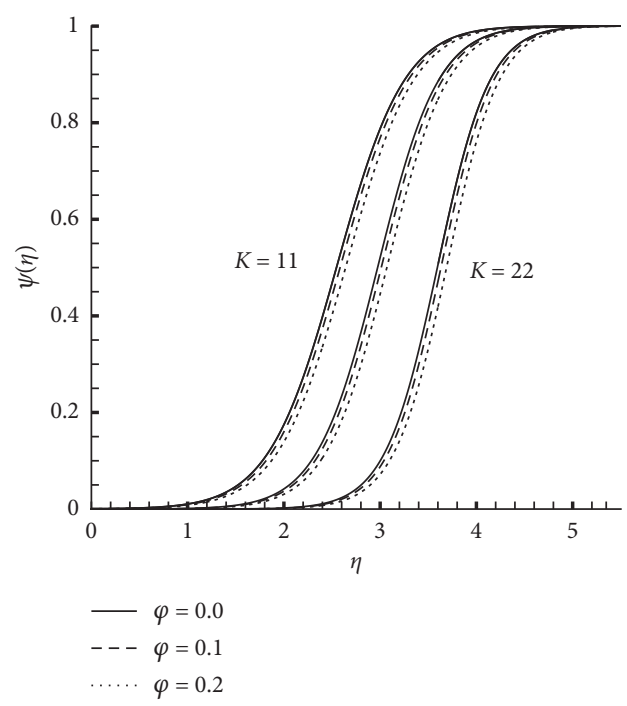

(a)

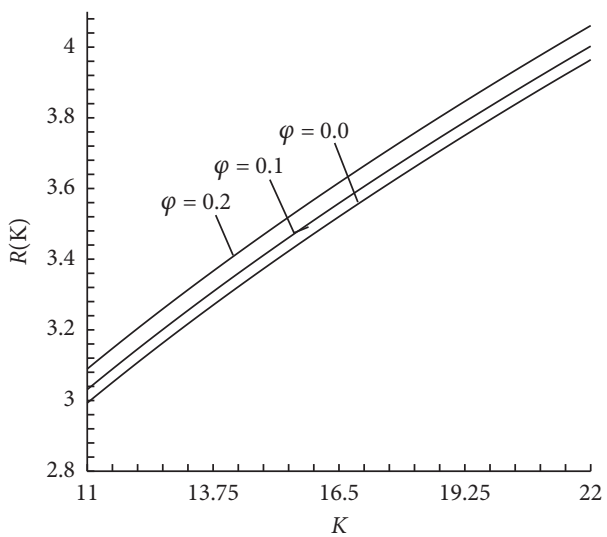

(b)

FIgURE 5: (a) Concentration profile $\psi(\eta)$ plotted against $\eta$ for increasing values of $K$ when $\operatorname{Sc}_{A}=1, \operatorname{Pr}=6.8, K_{s}=0.1, \phi=0.0, \phi=0.1$, and $\phi=0,2$; (b) a plot of $R(K)$, the position of the reaction front, for $K$ larger obtained from equation (27) for $\phi=0.0, \phi=0.1$, and $\phi=0.2$.

at $\eta=0$ does not match to that in equation (23). Therefore, they suggested to consider the condition at the inner edge of the reaction front where the inner solution $\psi_{0}$ decays exponentially small in $K$. As such, the following relations hold:

$$
\begin{aligned}
\zeta & =K^{-1} Y, \\
\psi & =\exp [K M(\zeta ; K)],
\end{aligned}
$$

where $\zeta<0$ is in the order of $O(1)$ for the region. Due to equation (28), it is readily known that $\psi(0)$ possesses exponentially decaying behaviors for large $K$. Therefore, we have

$$
\psi(0) \sim \exp \left(-M_{0} K\right), \quad \text { as } K \longrightarrow \infty, M_{0}=0.596 .
$$

Note that this relation still holds when the effect of nanofluids is considered.

It is worth mentioning to this end that the nanofluid is considered in our case; therefore, the nanoparticle volume fraction plays a role on $R(K)$. From equation (27), we notice that $R(K)$ is closely related to $\Delta$ which is equal to 0.647 , 0.685 , and 0.744 for $\phi=0.0, \phi=0.1$, and $\phi=0.2$, respectively. This indicates that $R(K)$ enhances as $\phi$ evolves for prescribed values of $K$, as shown in Figure 5(b).

\section{Autoignition}

Autoignition takes place at $K_{s}=0$. To decide the occurrence time of the autoignition, equation (10) subject to the following boundary conditions needs to be taken into account:

$$
\begin{aligned}
\psi^{\prime}(0) & =0, \\
\psi & \longrightarrow 1, \\
\text { as } \eta & \longrightarrow \infty .
\end{aligned}
$$

As shown in Figure 6(a), the nanoparticle volume fraction $\phi$ has perceptible effects on $\psi(0)$. For each $\phi$, there is a critical value of $K_{a}$. For $K>K_{a}$, two different branches are available when other parameters are prescribed. These solutions correspond to the two points in Figure 3, where $K_{s}=$ 0 for the case $\phi=0.1, \mathrm{Sc}_{A}=1$, and $\operatorname{Pr}=6.8$. Though not shown in the figures, it is expected that the lower and the upper branches for $\psi_{0}$ approach to 0 and 1, respectively, as $K$ approaches to infinity.

Particularly, for $K \longrightarrow \infty$, equation (10) suggests that the upper branch has the following form:

$$
\psi=1-K^{-1} \psi^{*}
$$

where $\psi^{*}$ is a scaling transformation function. If we let $\eta$ be unscaled, then the following leading order problem is derived:

$$
\psi^{* \prime \prime}+f \psi^{* 1}+\psi^{* 2}=0
$$

subject to the boundary conditions

$$
\begin{aligned}
\psi^{* \prime}(0) & =0, \\
\psi^{*} & \longrightarrow 1, \\
\text { as } \eta & \longrightarrow \infty .
\end{aligned}
$$

By solving this equation numerically, we obtain $\psi^{*}(0)=$ 0.833 for $\phi=0.0, \psi^{*}(0)=0.825$ for $\phi=0.1$, and $\psi^{*}(0)=$ 0.818 for $\phi=0.2$ when $\mathrm{Sc}_{A}=1$ and $\operatorname{Pr}=6.8$, respectively. As a result, we obtain the asymptotic expressions of $\psi(0)$ for $K \longrightarrow \infty$ as

$$
\begin{array}{ll}
\psi(0) \sim 1-0.833 K^{-1}+\cdots, & (\phi=0.0), \\
\psi(0) \sim 1-0.825 K^{-1}+\cdots, & (\phi=0.1), \\
\psi(0) \sim 1-0.818 K^{-1}+\cdots, & (\phi=0.2) .
\end{array}
$$

For example, asymptotic expression (35) is shown in Figure 6(a) by the dashed curve which is in good agreement 


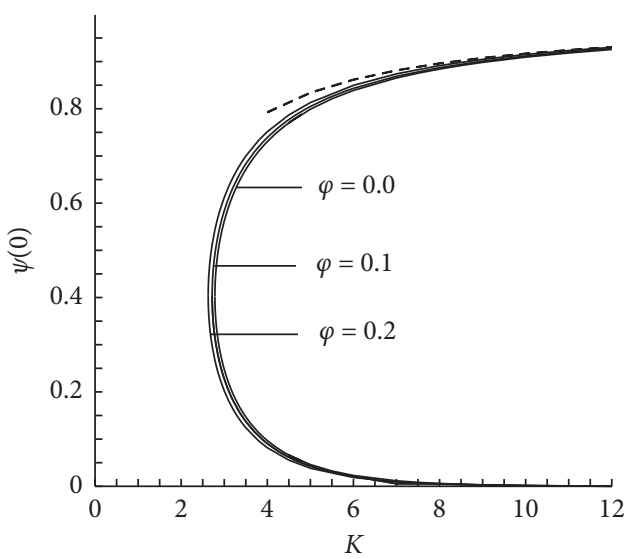

(a)

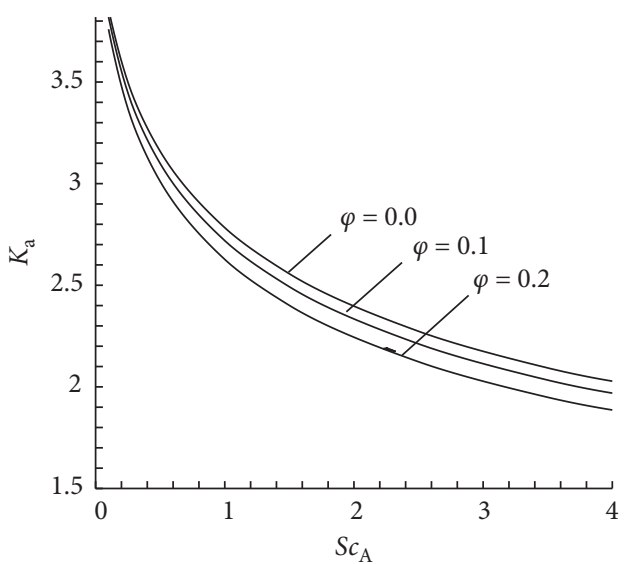

(b)

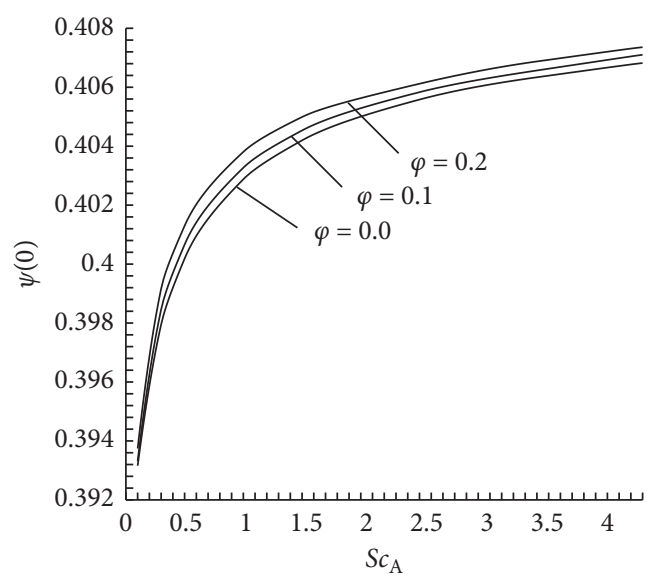

(c)

Figure 6: Autoignition: (a) a graph of $\psi(0)$ against $K$ obtained by solving equation (10) with boundary condition equation (30) for $\mathrm{Sc}_{A}=1$, $\operatorname{Pr}=6.8, \phi=0.0, \phi=0.1$, and $\phi=0.2$, (b) a graph of $K_{a}$ plotted against $\mathrm{Sc}_{A}$ for $\operatorname{Pr}=6.8, \phi=0.0, \phi=0.1$, and $\phi=0.2$, and (c) a graph of $\psi(0)$ when $K_{a}=K$ plotted against $\mathrm{Sc}_{A}$.

with the numerical solution, especially for large $K$. Also, from our previous analysis, the behavior of the lower solution branch becomes exponentially small as $K$ grows considerably large.

As expected, the critical value $K_{a}$ is dependent on $\mathrm{Sc}_{A}$ and $\phi$ as well. The dependence of $K_{a}$ on $\mathrm{Sc}_{A}$ is shown in Figure 6(b), with the corresponding values of $\psi(0)$ being shown in Figure 6(c). From these figures, we notice that $K_{a}$ reduces monotonously as $\mathrm{Sc}_{A}$ evolves. On the contrary, for prescribed values of $\mathrm{Sc}_{A}, K_{a}$ decreases gradually as $\mathrm{Sc}_{A}$ enlarges. Some critical values $K_{a}$ for various values of $\mathrm{Sc}_{A}$ are listed in Table 4 in which the comparison with the results of Chaudhary and Merkin [3] is presented, and good agreement is found.

\section{Solution Dependance on $\mathrm{Sc}_{A}$}

It is obvious that $\mathrm{Sc}_{A}$ can alter solution behaviours. We, therefore, discuss its influence on $\psi(\eta)$ when $\mathrm{Sc}_{A}$ is small and large, respectively.

5.1. Solution for Large $S c_{A}$. To check the significance of $\mathrm{Sc}_{A}$, we take $K$ and $K_{s}$ are both in the order of $O(1)$. From
TABLE 4: $K_{a}$ against $\mathrm{Sc}_{A}$ for various values of $\phi$ at $\operatorname{Pr}=6.8$.

\begin{tabular}{lcccc}
\hline $\mathrm{Sc}_{A}$ & & $\mathrm{Ka}$ & & $\begin{array}{c}\text { Chaudhary and Merkin [3] } \\
\phi=0.0\end{array}$ \\
& $\phi=0.0$ & $\phi=0.1$ & $\phi=0.2$ & \\
\hline 0.3 & 3.413 & 3.358 & 3.277 & 2.785 \\
0.5 & 3.1589 & 3.0989 & 3.0108 & \\
1 & 2.783 & 2.719 & 2.627 & \\
1.5 & 2.556 & 2.492 & 2.401 & \\
2.5 & 2.273 & 2.211 & 2.123 & \\
\hline
\end{tabular}

equation (10), it is known that solutions could be possible for large $\mathrm{Sc}_{A}$, provided that

$$
\begin{aligned}
\psi(\eta) & =1-\mathrm{Sc}_{A}^{-1 / 3} B(\xi), \\
\xi & =\mathrm{Sc}_{A}^{1 / 3} \eta,
\end{aligned}
$$

where $B(\xi)$ and $\xi$ are the scaling function and variable, respectively.

Equation (37) clearly shows that $\psi(0) \longrightarrow 1$ as $\mathrm{Sc}_{A}$ approaches to infinity. The reaction region therefore has the thickness of $\mathrm{O}\left(\mathrm{Sc}_{A}^{-1 / 3}\right)$ for considerably large $\mathrm{Sc}_{A}$. Substituting equation (37) in (10), it yields 


$$
B^{\prime \prime}+\left(\frac{C_{0}}{2} \xi^{2}-\mathrm{Sc}_{A}^{-1 / 3} \frac{\xi^{3}}{6}+\cdots\right) B^{\prime}+K B^{2}\left(1-\mathrm{Sc}_{A}^{-1 / 3} B\right)=0
$$

subject to the boundary conditions

$$
\begin{aligned}
B(0) & =-K_{s}\left[1-\mathrm{Sc}_{A}^{-1 / 3} B(0)\right], \\
B(\infty) & =0 .
\end{aligned}
$$

$B$ can be expanded, based on equations (38) and (39), as

$$
B\left(\xi, \mathrm{Sc}_{A}\right)=B_{0}(\xi)+\mathrm{Sc}_{A}^{-1 / 3} B_{1}(\xi) .
$$

From equation (37), we further obtain

$$
\psi(0)=1-\mathrm{Sc}_{A}^{-1 / 3} B_{0}(0)+\cdots, \quad \text { as } \mathrm{Sc}_{A} \longrightarrow \infty,
$$

which indicates that there is no possibility for the existence of multiple solutions at large $\mathrm{Sc}_{A}$ when $K$ and $K_{S}$ are both in the order of $O(1)$.

However, equation (41) will lose its valid range when $K_{s}$ and $K$ are not small. To handle this situation, we write

$$
\begin{aligned}
K_{s} & =\mathrm{Sc}_{A}^{1 / 3} K_{s}^{*}, \\
K & =\mathrm{Sc}_{A}^{-1 / 3} K^{*},
\end{aligned}
$$

where $K_{s}^{*}$ and $K^{*}$ both are $O(1)$ and $\xi=\operatorname{Sc}_{A} \eta$. Equation (10) is then reduced to the following leading order equation:

$$
\psi^{\prime \prime}+\frac{C_{0}}{2} \xi^{2} \psi \prime-K^{*} \psi(1-\psi)^{2}=0,
$$

subject to the boundary conditions

$$
\begin{aligned}
& \psi \prime(0)=K_{s}^{*} \psi(0), \\
& \psi(\infty)=1 .
\end{aligned}
$$

The hysteresis bifurcation point can then be calculated by solving equation (43). For example, we obtain $K_{s}^{*}=0.109$ and $K^{*}=3.220$ at $\phi=0.0, K_{s}^{*}=0.064$ and $K^{*}=2.568$ at $\phi=0.1$, and $K_{s}^{*}=0.039$ and $K^{*}=1.989$ at $\phi=0.2$ which further leads to

$$
\begin{aligned}
& K_{s}^{H} \sim 0.109 \mathrm{Sc}_{A}^{1 / 3}, \\
& K^{H} \sim 3.220 \mathrm{Sc}_{A}^{-1 / 3}, \\
& \mathrm{Sc}_{A} \longrightarrow \infty,
\end{aligned}
$$

when $\phi=0.0$.

$$
\begin{aligned}
& K_{s}^{H} \sim 0.064 \mathrm{Sc}_{A}^{1 / 3}, \\
& K^{H} \sim 2.568 \mathrm{Sc}_{A}^{-1 / 3}, \\
& \mathrm{Sc}_{A} \longrightarrow \infty,
\end{aligned}
$$

when $\phi=0.1$.

$$
\begin{aligned}
& K_{s}^{H} \sim 0.039 \mathrm{Sc}_{A}^{1 / 3}, \\
& K^{H} \sim 1.989 \mathrm{Sc}_{A}^{-1 / 3}, \\
& \mathrm{Sc}_{A} \longrightarrow \infty,
\end{aligned}
$$

when $\phi=0.2$.
As shown in Figure 7, the increase in $\mathrm{Sc}_{A}$ causes the decrease in $K^{H}$ (Figure $7(\mathrm{a})$ ) but the increase in $K_{s}^{H}$ (Figure $7(\mathrm{~b})$ ).

5.2. Solution for $S m a l l ~ S c_{A}$. In this case, the reaction region is thicker than the region of the boundary layer flow. Since the thick outer region is of extent $O\left(\mathrm{Sc}_{A}^{-1 / 2}\right)$, we introduce new variable $\varrho=\mathrm{Sc}_{A}^{1 / 2} \eta$ and leave $\psi(0)$ unscaled, and then write

$$
\psi\left(\varrho, \mathrm{Sc}_{A}\right)=\psi_{0}(\varrho)+\mathrm{Sc}_{A}^{1 / 2} \psi_{1}(\varrho)+\cdots .
$$

Substituting equation (46) into (10) and using the new scalings, at the leading order, we obtain

$$
\begin{aligned}
& \psi_{0}^{\prime \prime}+\varrho \psi_{0}^{\prime}-K \psi_{0}\left(1-\psi_{0}\right)^{2}=0, \\
& \psi_{0}(\infty)=1 .
\end{aligned}
$$

In the inner boundary layer region, we expand $\psi_{0}(\varrho)$ at the leading order, obtaining

$$
\psi_{0}(\varrho)=D_{0}+D_{1} \varrho+D_{2} \varrho^{2}+\cdots,
$$

where $D_{i}$ are constants which can be determined successively. Particularly, using equation (47), we obtain $D_{2}=(1 / 2)$ $K D_{0}\left(1-D_{0}\right)^{2}$.

At the order of $O\left(\operatorname{Sc}_{A}^{1 / 2}\right), \psi_{1}(\varrho)$ is linear and satisfies $\psi_{1}(\infty)=0$; we therefore expand it, as $\varrho$ approaches to 0 , by

$$
\psi_{1}=E_{0}+E_{1} \varrho+\cdots,
$$

where $E_{i}$ are constants.

On the contrary, based on equations (48) and (49), it suggests

$$
\psi\left(\eta, \mathrm{Sc}_{A}\right)=\psi_{0}(\eta)+\mathrm{Sc}_{A}^{1 / 2} \psi_{1}(\eta)+\cdots
$$

Note that the inner region has to satisfy the boundary conditions (8b) at $\eta=0$. Therefore, the leading order equation gives

$$
\psi_{0}^{\prime \prime}=0,
$$

subject to the boundary conditions

$$
\begin{aligned}
& \psi_{0}^{\prime}=K_{s} \psi_{0}(0), \\
& \psi_{0} \longrightarrow D_{0}, \\
& \text { as } \eta \longrightarrow \infty
\end{aligned}
$$

The solution of this equation is $D_{0}=0$. At the order of $O\left(\mathrm{Sc}_{A}^{1 / 2}\right)$, it holds

$$
\psi_{1}^{\prime \prime}=0,
$$

subject to the boundary conditions

$$
\begin{aligned}
& \psi_{1}^{\prime}(0)=K_{s} \psi_{1}(0), \\
& \psi_{1} \sim D_{1} \eta+E_{1}, \\
& \text { as } \eta \longrightarrow \infty,
\end{aligned}
$$

which give $E_{1}=D_{1} / K_{s}$. As a result, we obtain

$$
\psi_{1}=D_{1}\left(\frac{\eta+1}{K_{s}}\right) .
$$

Thus, the appropriate inner condition for equation (47) is

$$
\psi_{0}(0)=0 .
$$

The solutions of equation (47) subject to the inner condition (56) determine that $D_{1}=\psi_{0}^{\prime}(0)$ from which we obtain 


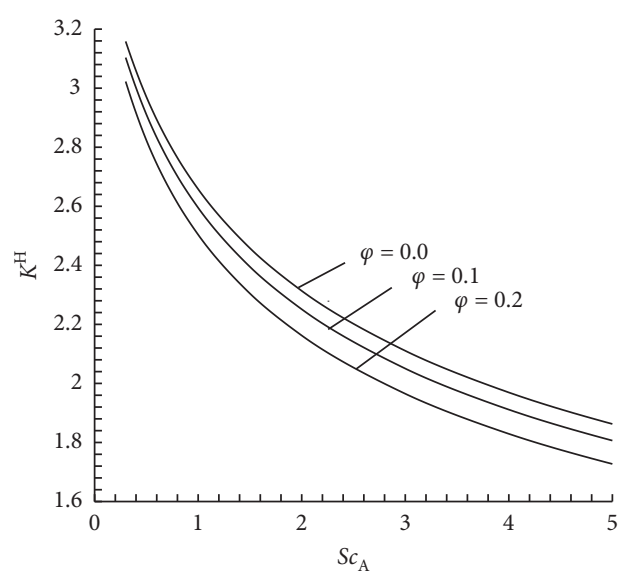

(a)

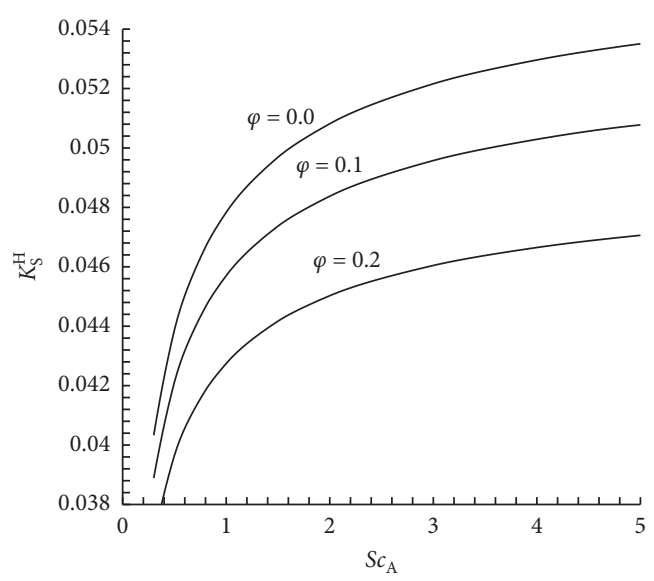

(b)

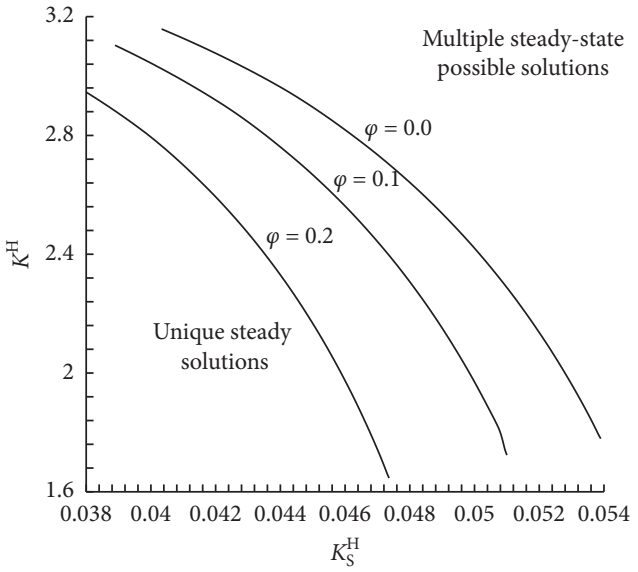

(c)

Figure 7: Graphs of (a) $K^{H}$ plotted against $\mathrm{Sc}$ for $\operatorname{Pr}=6.8, \phi=0.0, \phi=0.1$, and $\phi=0.2$, (b) $K_{S}^{H}$ plotted against $\mathrm{Sc}$ for $\operatorname{Pr}=6.8, \phi=0.0$, $\phi=0.1$, and $\phi=0.2$, and (c) $K^{H}$ plotted against $K_{S}^{H}$, the region where multiple steady-state solutions are possible are also indicated.

$$
\psi(0) \sim \frac{D_{1}}{K_{s}} \mathrm{Sc}_{A}^{1 / 2}+\ldots, \quad \operatorname{as~Sc}_{A} \longrightarrow 0
$$

Equation (57) indicates that there is a singularity at $K_{s}=0$. To determine the nature of the hysteresis bifurcation for small $\mathrm{Sc}_{A}$, we put

$$
K_{s}=K_{s}^{*} \mathrm{Sc}_{A}^{1 / 2} .
$$

The solution at the leading order is simplified as

$$
\psi_{0}(\eta)=D_{0} .
$$

However, at the order of $O\left(\mathrm{Sc}_{A}^{1 / 2}\right)$, equation (53) still holds and satisfies

$$
\begin{aligned}
& \psi_{1}^{\prime}(0)=K_{s} \psi_{0}(0), \\
& \psi_{1} \sim D_{1} \eta+E_{1}, \\
& \text { as } \eta \longrightarrow \infty,
\end{aligned}
$$

which give $D_{1}=K_{s}^{*} D_{0}$. So, we have

$$
\psi_{1}=K_{s}^{*} D_{0} \eta+E_{1}
$$

To balance the outer region with the inner boundary condition, we obtain

$$
\psi\left(\varrho, \mathrm{Sc}_{A}\right) \sim D_{0}+K_{s}^{*} D_{0} \varrho+\mathrm{Sc}_{A}^{1 / 2} E_{1}+\cdots, \quad \text { as } \varrho \longrightarrow 0 .
$$

We, therefore, are able to find the solution of equation (46) by solving leading order equation (47) subject to the inner boundary condition

$$
\psi_{0}^{\prime}=K_{s}^{*} \psi_{0}, \quad \text { on } \varrho \longrightarrow 0 .
$$

It is now expected that equation (47) subject to the inner boundary conditions (63) has multiple solutions. The hysteresis bifurcated point can be determined in a similar way. We obtain the critical values $K_{s}^{* H}=0.129$ and $K^{H}=4.712$ for $\phi=0.0, K_{s}^{* H}=0.102$ and $K^{H}=4.311$ for $\phi=0.1$, and $K_{s}^{* H}=0.080$ and $K^{H}=3.881$ for $\phi=0.2$, which further give 


$$
\begin{array}{rl}
K^{H} & \longrightarrow 4.712, \\
K_{s}^{H} & \sim 0.129 \mathrm{Sc}_{A}^{1 / 2}, \\
\text { as } \mathrm{Sc}_{A} & \longrightarrow 0, \\
\text { when } \phi & =0.0, \\
K^{H} & \longrightarrow 4.311, \\
K_{s}^{H} & \sim 0.102 \mathrm{Sc}_{A}^{1 / 2}, \\
\text { as Sc } & \longrightarrow 0, \\
\text { when } \phi^{1 / 2} & 0.1, \\
K^{H} & \longrightarrow 3.881, \\
K_{s}^{H} & \sim 0.080 \mathrm{Sc}_{A}^{1 / 2}, \\
\text { as Sc } & \longrightarrow 0, \\
\text { when } \phi & =0.2 .
\end{array}
$$

\section{Conclusions}

The homogeneous-heterogeneous reactions in a nanofluid with the suspended single-walled carbon nanotubes at the stagnation-point region of a plane surface have been investigated. The homogeneous (bulk) reaction is assumed to be isothermal cubic autocatalytic, while the heterogeneous (surface) reaction is single, isothermal, and first order. The homogeneous nanofluid model is employed with equal diffusion coefficients being considered for both reactants and autocatalysts. By doing similarity transformation, multiple solutions of the model have been captured for particular sets of physical parameters, which are overlooked in previous publications on modelling homogeneous-heterogeneous reactions. Furthermore, we have discovered the limitation of Magyari's conclusion [25] about that the solutions of the homogeneous nanofluid model can be recovered from those of the regular fluid model. This statement is only valid for a few special situations.

In summary, the main novel aspects of this study are as follows:

(i) Multiple solutions for modelling homogeneousheterogeneous reactions are found for some sets of physical parameters, and their origins are discussed

(ii) Homogeneous and heterogeneous reaction rates $K$ and $K_{s}$, which are the obvious key factors to affect the chemical reactions, are important parameters to give multiple solutions

(iii) Nanoparticle volume fraction $\phi$ plays a significant role on altering solution stability, and it also can affect the stability of chemical reactions

(iv) Magyari's conclusion [25] on that the solutions of the homogeneous nanofluid model can be recovered from those of the regular fluid model is found to be limited and only valid for a few special cases

\section{Data Availability}

The data used to support the findings of this study are included within the article.

\section{Conflicts of Interest}

The authors declare that they have no conflicts of interest.

\section{Acknowledgments}

This work was partially supported by the National Natural Science Foundation of China (Grant no. 11872241). This work was supported in part by the Australian Research Council through the Centre of Excellence grant CE140100003 to QS.

\section{References}

[1] W. R. Williams, M. T. Stenzel, X. Song, and L. D. Schmidt, "Bifurcation behavior in homogeneous-heterogeneous combustion: I. Experimental results over platinum," Combustion and Flame, vol. 84, no. 3-4, pp. 277-291, 1991.

[2] X. Song, W. R. Williams, L. D. Schmidt, and R. Aris, "Bifurcation behavior in homogeneous-heterogeneous combustion: II. Computations for stagnation-point flow," Combustion and Flame, vol. 84, no. 3-4, pp. 292-311, 1991.

[3] M. A. Chaudhary and J. H. Merkin, "A simple isothermal model for homogeneous-heterogeneous reactions in boundary-layer flow. I equal diffusivities," Fluid Dynamics Research, vol. 16, no. 6, pp. 311-333, 1995.

[4] M. A. Chaudhary and J. H. Merkin, "A simple isothermal model for homogeneous-heterogeneous reactions in boundary-layer flow. II different diffusivities for reactant and autocatalyst," Fluid Dynamics Research, vol. 16, no. 6, pp. 335-359, 1995.

[5] H. Xu, "A homogeneous-heterogeneous reaction model for heat fluid flow in the stagnation region of a plane surface," International Communications in Heat and Mass Transfer, vol. 87, pp. 112-117, 2017.

[6] S. U. S. Choi, Z. G. Zhang, W. Yu, F. E. Lockwood, and E. A. Grulke, "Anomalous thermal conductivity enhancement in nanotube suspensions," Applied Physics Letters, vol. 79, no. 14 , pp. 2252-2254, 2001.

[7] F. D. S. Marquis and L. P. F. Chibante, "Improving the heat transfer of nanofluids and nanolubricants with carbon nanotubes," JOM, vol. 57, no. 12, pp. 32-43, 2005.

[8] R. K. Tiwari and M. K. Das, "Heat transfer augmentation in a two-sided lid-driven differentially heated square cavity utilizing nanofluids," International Journal of Heat and Mass Transfer, vol. 50, no. 9-10, pp. 2002-2018, 2007.

[9] N.-L. Xu, H. Xu, and A. Raees, "Homogeneous-heterogeneous reactions in flow of nanofluids near the stagnation region of a plane surface: the Buongiorno's model," International Journal of Heat and Mass Transfer, vol. 125, pp. 604-609, 2018.

[10] H. Xu, "Homogenous-heterogeneous reactions of Blasius flow in a nanofluid," Journal of Heat Transfer-Transactions of the ASME, vol. 141, no. 2, Article ID 024501, 2019.

[11] J. Buongiorno, "Convective transport in nanofluids," Journal of Heat Transfer, vol. 128, no. 3, pp. 240-250, 2006.

[12] T. Hayat, S. Ahmed, T. Muhammad, A. Alsaedi, and M. Ayub, "Computational modeling for homogeneous-heterogeneous 
reactions in three-dimensional flow of carbon nanotubes," Results in Physics, vol. 7, pp. 2651-2657, 2017.

[13] N. Bachok, A. Ishak, and I. Pop, "On the stagnation-point flow towards a stretching sheet with homogeneous-heterogeneous reactions effects," Communications in Nonlinear Science and Numerical Simulation, vol. 16, no. 11, pp. 4296-4302, 2011.

[14] M. Sheikh and Z. Abbas, "Homogeneous-heterogeneous reactions in stagnation point flow of Casson fluid due to a stretching/shrinking sheet with uniform suction and slip effects," Ain Shams Engineering Journal, vol. 8, no. 3, pp. 467-474, 2017.

[15] P. K. Kameswaran, S. Shaw, P. Sibanda, and P. V. S. N. Murthy, "Homogeneous-heterogeneous reactions in a nanofluid flow due to a porous stretching sheet," International Journal of Heat and Mass Transfer, vol. 57, no. 2, pp. 465-472, 2013.

[16] T. Hayat, M. I. Khan, A. Alsaedi, and M. I. Khan, "Homogeneous-heterogeneous reactions and melting heat transfer effects in the MHD flow by a stretching surface with variable thickness," Journal of Molecular Liquids, vol. 223, pp. 960968, 2016.

[17] T. Hayat, M. Farooq, and A. Alsaedi, "Homogeneous-heterogeneous reactions in the stagnation point flow of carbon nanotubes with Newtonian heating," AIP Advances, vol. 5, no. 2, Article ID 027130, 2015.

[18] S. Iijima, "Helical microtubules of graphitic carbon," Nature, vol. 354 , no. 6348 , pp. 56-58, 1991.

[19] D. S. Bethune, C. H. Kiang, M. S. de Vries et al., "Cobaltcatalysed growth of carbon nanotubes with single-atomiclayer walls," Nature, vol. 363, no. 6430, pp. 605-607, 1993.

[20] R. Ramasubramaniam, J. Chen, and H. Liu, "Homogeneous carbon nanotube/polymer composites for electrical applications," Applied Physics Letters, vol. 83, no. 14, pp. 2928-2930, 2003.

[21] Q. Z. Xue, "Model for thermal conductivity of carbon nanotube-based composites," Physica B: Condensed Matter, vol. 368, no. 1-4, pp. 302-307, 2005.

[22] X. Ma, F. Su, J. Chen, T. Bai, and Z. Han, "Enhancement of bubble absorption process using a CNTs-ammonia binary nanofluid," International Communications in Heat and Mass Transfer, vol. 36, no. 7, pp. 657-660, 2009.

[23] H. H. Khoshmehr, A. Saboonchi, and M. B. Shafii, "The quenching of silver rod in boiling carbon nano tube-water nanofluid," International Journal of Thermal Sciences, vol. 75, pp. 95-104, 2014.

[24] S. S. Park and N. J. Kim, "A study on the characteristics of carbon nanofluid for heat transfer enhancement of heat pipe," Renewable Energy, vol. 65, pp. 123-129, 2014.

[25] E. Magyari, "Comment on the homogeneous nanofluid models applied to convective heat transfer problems," Acta Mechanica, vol. 222, no. 3-4, pp. 381-385, 2011.

[26] L. Rosenhead, Laminar Boundary Layers, Clarendon Press, Oxford, UK, 1963.

[27] A. Granas, R. B. Guenther, and J. W. Lee, "The shooting method for the numerical solution of a class of nonlinear boundary value problems," SIAM Journal on Numerical Analysis, vol. 16, no. 5, pp. 828-836, 1979.

[28] J. Billingham and D. J. Needham, "A note on the properties of a family of travelling-wave solutions arising in cubic autocatalysis," Dynamics and Stability of Systems, vol. 6, no. 1, pp. 33-49, 1991. 